\title{
The Riccati Equation: Pinching of Forcing and Solutions
}

\author{
Marlies Gerber, Boris Hasselblatt, and Daniel Keesing
}

\section{CONTENTS}

1. Statement of Results

2. Motivation

3. Proofs

4. Application: Proof of Theorem 1.6

5. Explicit Solutions and Polynomial Asymptotics

6. Numerical Work and Open Questions

Acknowledgments

References
2000 AMS Subject Classification: Primary: 34H05, 34D05, 34A99; Secondary: 37D40

Keywords: Riccati equation, foliations, filtering, control
A problem at the interface of differential geometry and dynamical systems gives rise to the question of what control of solutions of the Riccati equation $\dot{x}+x^{2}=k(t)$ with positive right-hand side can be obtained from control of the forcing term $k$. We show that a known result about "relative" pinching is optimal and refine two known theorems. This gives improved regularity of horospheric foliations and may be of interest in control or filtering theory.

\section{STATEMENT OF RESULTS}

The differential equations $\dot{x}=\alpha x^{2}+\beta t^{n}$ and $\dot{x}=\alpha x^{2}+$ $\beta t+\gamma t^{2}$ as well as, more generally, $\dot{x}=\alpha(t) x^{2}+\beta(t) x+$ $\gamma(t)$, are called Riccati (differential) equations. (If $\alpha \neq 0$, the latter reduces to $\dot{y}=y^{2}+\eta(t)$ by writing $x=(y / \alpha)-$ $\left.(\beta / 2 \alpha)-\left(\alpha^{\prime} / 2 \alpha^{2}\right).\right)$

Matrix forms of the Riccati differential equation as well as algebraic counterparts thereof are useful for applied mathematics, especially filtering and control problems, and this has motivated a large body of research; see, for example [Bittanti et al. 91]. A matrix form of $\dot{x}+x^{2}=k(t)$ is of interest in differential geometry, especially with respect to asymptotic spectral information about solutions. This is our principal interest here, and [Hasselblatt 94b] explains how to obtain such spectral information from the study of solutions of the scalar equation $\dot{x}+x^{2}=k(t)$, which we undertake.

Two known theorems provide our starting point.

Theorem 1.1. If $K_{1}>K_{2}>0$ and $x_{i}>0$ solves $\dot{x}+x^{2}=$ $k_{i}(t)$ for $i=1,2$, where $K_{2} \leq k_{2}(t) \leq k_{1}(t) \leq K_{1}$ and $x_{1}(0) \leq \sqrt{K_{1}}, x_{2}(0) \geq \sqrt{K_{2}}$, then $x_{2}(t) \geq \sqrt{K_{2}}$ and $x_{1}(t) \leq \sqrt{K_{1}}$ for all $t>0$.

In particular, if $a \in(0,1)$ and $K_{2}>a K_{1}$, then $x_{2}(t)>$ $\sqrt{a} x_{1}(t)$ for all $t>0$. 
This is sharp, because if the $k_{i}$ are constant, then $x_{i}(t)=\sqrt{k_{i}}$ are solutions. Theorem 1.1 has applications to differential geometry for which it suffices to control the ratio of solutions for large $t$ [Hasselblatt 94b]. Therefore, one would like to control the ratio of solutions via the ratio of the right-hand sides (and maybe the ratio of initial conditions, but this is not important for those applications).

Theorem 1.2. ([Hasselblatt 94b]) If $a \in(0,1), 0<$ $a k_{1}(t)<k_{2}(t)<k_{1}(t)$, and $x_{i}$ is the solution of $\dot{x}+x^{2}=$ $k_{i}(t)$ for $i=1,2$, then

$x_{2}(0)>a x_{1}(0)>0$ implies $x_{2}(t)>a x_{1}(t)$ for all $t>0$.

Analogy with Theorem 1.1 suggests that Theorem 1.2 should hold with $a$ replaced by $\sqrt{a}$ in (1-1), and Proposition 5.1 provides instances in which an asymptotic version of this is true. However, we show that Theorem 1.2 is sharp and that additional hypotheses on the initial condition do not help. Moreover, we establish that even a weaker version of Theorem 1.2 that would suffice for the applications to differential geometry is sharp:

Theorem 1.3. Theorem 1.2 is sharp: One cannot replace $a$ in the conclusion by $a^{H}$ for any $H<1$, even under more stringent hypotheses on the initial conditions. Specifically, if $0<a<c<1$ and $y_{1}, y_{2}>0$, then there exist $k_{1}(t), k_{2}(t)$ with $0<a k_{1}(t)<k_{2}(t)<k_{1}(t) \leq 1$ such that the solutions $x_{i}$ of $\dot{x}+x^{2}=k_{i}(t)$ with $x_{i}(0)=y_{i}$ satisfy $x_{2}(T)<c x_{1}(T)$ for arbitrarily large $T$.

Combining the hypotheses of Theorem 1.1 and Theorem 1.2 nevertheless leads to a common refinement:

Theorem 1.4. Suppose $0 \leq b \leq a \leq 1, K_{1}>K_{2}>b K_{1}>$ 0 and $K_{2} \leq k_{2}(t) \leq k_{1}(t) \leq K_{1}$ (absolute b-pinching) as well as $0<a k_{1}(t)<k_{2}(t)<k_{1}(t)^{1}$ for all $t \geq 0$ (relative a-pinching). Let

$$
B(a, b):=\frac{1}{2}\left(a-b+\sqrt{(a+b)^{2}+4(1-a) b}\right) .
$$

If $x_{i}$ is a solution of $\dot{x}+x^{2}=k_{i}(t)$ for $i=1,2$ with $x_{2}(0)>B(a, b) x_{1}(0)>0$ and $x_{1}(0) \leq \sqrt{K_{1}}, x_{2}(0) \geq$ $\sqrt{K_{2}}$, then $x_{2}(t)>B(a, b) x_{1}(t)$ for all $t>0$.

Also, if $H:=1 /(b+1) \in[1 / 2,1]$ and $x_{i}$ is a solution of $\dot{x}+x^{2}=k_{i}(t)$ for $i=1,2$ with $x_{2}(0)>a^{H} x_{1}(0)$ and $x_{1}(0) \leq \sqrt{K_{1}}, x_{2}(0) \geq \sqrt{K_{2}}$, then $x_{2}(t)>a^{H} x_{1}(t)$ for all $t>0$.

\footnotetext{
${ }^{1}$ This is to be interpreted as $k_{1}(t)=k_{2}(t)$ when $a=1$.
}

Remark 1.5. This refines both previous results because $B(a, b) \geq a$ (with strict inequality for $b>0, a<1$ ) and $B(a, a)=\sqrt{a}$.

Theorem 1.4 can be applied to geodesic flows to give Theorem 4.3 and hence:

Theorem 1.6. For $0 \leq b \leq a \leq 1$, a compact b-pinched relatively a-pinched Riemannian manifold has $C^{a-b+\sqrt{(a+b)^{2}+4(1-a) b}}$ (and hence, $C^{2 a^{1 /(b+1)}}$ ) horospheric foliations.

\section{MOTIVATION}

The need for asymptotic pinching of the solutions of Riccati equations arises in the context of free particle motion (the geodesic flow) on a Riemannian manifold of negative sectional curvature, which provides the primary example of a classical mechanical system that is ergodic (and indeed chaotic). These properties are established using hyperbolicity, i.e., that any two orbits in phase space drift apart with a uniform exponential rate, either in the past or in the future, and usually both [Katok and Hasselblatt 95]. Ergodicity is established by an argument due to Hopf [Katok and Hasselblatt 95, page 217], which uses the stable and unstable foliations [Hopf 39], [Katok and Hasselblatt 95, Theorem 5.4.16] and requires some regularity of these $\left(C^{1}\right.$ in his original work about geodesic flows on surfaces [Hopf 39], absolute continuity via a Hölder condition in later work by Anosov and Sinai [Anosov and Sinai 67]).

This regularity is obtained using relations between the fastest and slowest possible rates of orbit separation (see, e.g., [Hasselblatt and Wilkinson 99]), which are in turn connected with variations in sectional curvature [Hirsch and Pugh 75], [Klingenberg 82, Theorem 3.2.17], [Hasselblatt 94a]. These results use the following line of reasoning. The linearized geodesic flow is described by the Jacobi equation, which transforms into the Riccati equation by changing to variables more closely related to the expanding and contracting directions (see the beginning of Section 5 and [Paternain 99, page 37]). The right-hand side of the Riccati equation involves sectional curvature, and absolute a-pinching, $\max \mid$ curvature $|>a \min |$ curvature $\mid$, gives upper and lower bounds for the right-hand side. Theorem 1.1 produces asymptotic bounds of solutions (absolute bunching); having these is known to imply a corresponding degree of regularity of the invariant foliations (see, e.g., [Fenichel 72, Hirsch et al. 77]). 
Instead of absolute bunching, it suffices to assume relative bunching to obtain regularity of the invariant foliations [Hasselblatt 94a]. In the present context, absolute bunching corresponds to bounds on the solutions of the Riccati equation. In general, it gives control of some ratio of bounds on contraction and expansion rates. Relative bunching corresponds to bounding a ratio of solutions of the Riccati equation instead. In terms of the dynamical system, it means that one need not control the ratio of the largest expansion rate in the system and the smallest expansion rate in the system, but only the ratio of largest and smallest rates at each point in phase space.

This raises the question of whether pointwise control of sectional curvatures implies pointwise control of expansion rates. Does relative a-pinching $\left(\max _{\text {at } p} \mid\right.$ curvature $\left|>a \min _{\text {at } p}\right|$ curvature $\mid$ for every $p$ ) imply a corresponding degree of relative bunching? This question was addressed in [Hasselblatt 94b] with an affirmative answer, but with less bunching than obtained from absolute pinching. The difference corresponds exactly to the difference between Theorem 1.1 and Theorem 1.2, and, by Theorem 1.3, Theorem 1.2 cannot be improved to include a square root as in Theorem 1.1. This does not rule out an analogous conjecture for relations between relative pinching and relative bunching, because it is not clear to which extent a relatively $a$ pinched manifold can fail to be absolutely $a$-pinched.

\section{PROOFS}

Proof of Theorem 1.1: If $t$ is such that $x_{2}(t) \leq \sqrt{K_{2}}$, then $\dot{x}_{2}(t)=k_{2}(t)-x_{2}^{2}(t) \geq 0$, so $x_{2}(t) \geq \sqrt{K_{2}}$ for all $t>0$. Likewise, $x_{1}(t) \leq \sqrt{K_{1}}$ for all $t>0$.

Proof of Theorem 1.2: Suppose $x_{2}(t) \leq a x_{1}(t)$. Then (suppressing $t$-dependence)

$$
\begin{aligned}
\dot{x}_{2} x_{1}-x_{2} \dot{x}_{1} & =\left(k_{2}-x_{2}^{2}\right) x_{1}-x_{2}\left(k_{1}-x_{1}^{2}\right) \\
& >\left(a x_{1}-x_{2}\right) k_{1}+x_{1} x_{2}\left(x_{1}-x_{2}\right) \\
& >\left(a x_{1}-x_{2}\right)\left(k_{1}+x_{1} x_{2}\right) \geq 0
\end{aligned}
$$

which implies $\frac{d}{d t} \frac{x_{2}}{x_{1}}>0$. Therefore, $x_{2}>a x_{1}$ for all $t>0$.

The last strict inequality is not sharp because it results from replacing $\left(x_{1}-x_{2}\right)$ by $\left(a x_{1}-x_{2}\right)$. Accordingly, [Hasselblatt $94 \mathrm{~b}$, page 62 , line 12] conjectures that if $0<$ $a k_{1}(t)<k_{2}(t)<k_{1}(t)$ and $x_{i}$ is the solution of $\dot{x}+x^{2}=$ $k_{i}(t)$ for $i=1,2$, then $x_{2}(0)>\sqrt{a} x_{1}(0)$ implies $x_{2}(t)>$ $\sqrt{a} x_{1}(t)$ for all $t>0$.
Proof of Theorem 1.3: Take $a<a^{\prime}<c^{\prime}<c, M>0$ such that $c^{\prime}+(4 / M)<c, \epsilon>0$ such that $(M+2)^{2} \epsilon^{2}<$ $1 / 2, a^{\prime}<c^{\prime}\left(1-(M+2)^{2} \epsilon^{2}\right)$ and $a^{\prime} k_{1}(t)=k_{2}(t)$ (then $\left.a k_{1}(t)<k_{2}(t)\right)$. If $k>0$, then $\sqrt{k}$ is an equilibrium of the autonomous ordinary differential equation $\dot{x}+x^{2}=k$ that attracts all positive solutions. Thus, there is a $\tau>1$ such that choosing $k_{1}(t)=\epsilon / 2$ on $[0, \tau]$ gives $x_{i}(\tau)<\epsilon$ for $i=1,2$. Now take $k_{1}(t)=1$ for $t \geq \tau+\epsilon$ and increasing on $[\tau, \tau+\epsilon]$. Let $T=\tau+(M+1) \epsilon$.

The Riccati equation implies that $\dot{x}_{1}(t) \leq k_{1}(t) \leq 1$ for $t \geq \tau$, so $x_{1}(t) \leq \epsilon+(t-\tau) \leq(M+2) \epsilon$ for $t \in[\tau, T]$, which implies via the Riccati equation that $\dot{x}_{1}(t) \geq 1-$ $(M+2)^{2} \epsilon^{2}$ for $t \in[\tau+\epsilon, T]$, hence

$$
x_{1}(T) \geq M \epsilon\left(1-(M+2)^{2} \epsilon^{2}\right) \text {. }
$$

Meanwhile, $\dot{x}_{2}(t) \leq k_{2}(t) \leq a^{\prime}$ for $t \geq \tau$, hence $x_{2}(T) \leq \epsilon+a^{\prime}(M+1) \epsilon$ and

$$
\begin{aligned}
\frac{x_{2}(T)}{x_{1}(T)} & \leq \frac{\epsilon+a^{\prime}(M+1) \epsilon}{M \epsilon\left(1-(M+2)^{2} \epsilon^{2}\right)} \\
& =\frac{a^{\prime}}{1-(M+2)^{2} \epsilon^{2}}+\frac{a^{\prime}+1}{M\left(1-(M+2)^{2} \epsilon^{2}\right)} \\
& <c^{\prime}+\frac{2}{M\left(1-(M+2)^{2} \epsilon^{2}\right)}<c^{\prime}+\frac{4}{M}<c .
\end{aligned}
$$

Applying this argument repeatedly yields infinitely many $T>0$ with this property that are at least 1 apart: Take $k_{1}$ decreasing to $\epsilon / 2$ on $[T, T+\epsilon]$ and restart the argument with initial values $x_{i}(T+\epsilon)$.

Proof of Theorem 1.4: If $0<r \leq B(a, b)$, then $f(r):=$ $r \frac{r-a}{1-r} \leq b$ by the quadratic formula because $f$ is increasing on $[a, B(a, b)]$. If $x_{2}(t) \leq r x_{1}(t)$, then (suppressing $t$ )

$$
\begin{aligned}
\dot{x}_{2} x_{1}-x_{2} \dot{x}_{1} & =\left(k_{2}-x_{2}^{2}\right) x_{1}-x_{2}\left(k_{1}-x_{1}^{2}\right) \\
& >\left(a x_{1}-x_{2}\right) k_{1}+x_{1} x_{2}\left(x_{1}-x_{2}\right) \\
& \geq\left(\frac{a}{r}-1\right) x_{2} k_{1}+x_{1} x_{2}^{2}\left(\frac{1}{r}-1\right) \\
& \geq(a-r) \frac{x_{2} k_{1}}{r}+\frac{x_{2}^{3}}{r}\left(\frac{1}{r}-1\right) \\
& \geq \frac{x_{2}}{r}\left(\frac{1}{r}-1\right)\left[x_{2}^{2}-b k_{1}\right] \geq 0,
\end{aligned}
$$

by Theorem 1.1, which implies $\frac{d}{d t} \frac{x_{2}}{x_{1}}>0$. Therefore, $x_{2}>r x_{1}$ for all $t>0$.

The second claim reduces to showing that $f\left(a^{1 /(b+1)}\right) \leq b$, which follows from $f\left(a^{\alpha}\right) \leq(1 / \alpha)-1$, hence from $g(x):=x^{-\alpha}-1-\alpha x^{-\alpha}+\alpha-\alpha x^{\alpha}+\alpha x \geq 0$ for $x \in[0,1]$. This holds because $g^{\prime}(x)=$ $\alpha(\alpha-1) x^{-\alpha-1}-\alpha^{2} x^{\alpha-1}+\alpha$, so $g(1)=g^{\prime}(1)=0$ and $g^{\prime \prime}(x)=\alpha\left(1-\alpha^{2}\right) x^{-\alpha-2}+\alpha^{2}(1-\alpha) x^{\alpha-2} \geq 0$ for $x \in[0,1]$. 


\section{APPLICATION: PROOF OF THEOREM 1.6}

Theorem 1.1 and Theorem 1.2 arose as part of the proof of a corresponding result in differential geometry. Theorem 1.1 is a step in the proof of hyperbolicity of the geodesic flow of a manifold of negative curvature [Anosov and Sinai 67, Klingenberg 82] and gives "absolute bunching" information from absolute pinching of sectional curvature. A refined argument that obtains bunching information (as in Definition 4.2) from relative pinching of sectional curvature proved Theorem 1.2 along the way [Hasselblatt 94b]. Theorem 1.4 can be inserted in the corresponding place in the proof of the results in [Hasselblatt $94 \mathrm{~b}]$ to refine those results.

Definition 4.1. The sectional curvature of a compact negatively curved Riemannian manifold $N$ is relatively a-pinched if $C \leq$ sectional curvature $<a C$ for some $C: N \rightarrow-\mathbb{R}_{+}$. If $C$ is constant, the curvature is said to be (absolutely) $a$-pinched.

Definition 4.2. A flow $\varphi^{t}$ on a compact Riemannian manifold $M$ is said to be an Anosov flow with Anosov splitting $\left(E^{u}, E^{s}\right):=\left(E^{s u} \oplus E^{\varphi}, E^{s s} \oplus E^{\varphi}\right)$ if $T M=E^{s u} \oplus E^{s s} \oplus E^{\varphi}$, $E^{\varphi}=\operatorname{span}\{\dot{\varphi}\} \neq\{0\}$ and $\exists \lambda<1, C>0, \forall p \in M, t>0$

$$
\begin{aligned}
& \left\|D \varphi^{t}(v)\right\| \leq C \lambda^{t}\|v\|\left(v \in E^{s}(p)\right) \text { and } \\
& \left\|D \varphi^{-t}(u)\right\| \leq C \lambda^{t}\|u\|\left(u \in E^{u}(p)\right) .
\end{aligned}
$$

Say that $\varphi^{t}$ is $\alpha$-bunched if there exist $\mu_{f} \leq \mu_{s}<1<$ $\nu_{s} \leq \nu_{f}: M \times \mathbb{R}_{+} \rightarrow \mathbb{R}_{+}$with

$$
\begin{aligned}
& \lim _{t \rightarrow \infty} \sup _{p \in M} \mu_{s}(p, t) \nu_{s}(p, t)^{-1} \mu_{f}(p, t)^{-\alpha}=0, \\
& \lim _{t \rightarrow \infty} \sup _{p \in M} \mu_{s}(p, t) \nu_{s}(p, t)^{-1} \nu_{f}(p, t)^{\alpha}=0
\end{aligned}
$$

such that for all $p \in M, v \in E^{s s}(p), u \in E^{s u}\left(\varphi^{t} p\right), t>0$, we have

$$
\begin{gathered}
\mu_{f}(p, t)\|v\| \leq\left\|D \varphi^{t}(v)\right\| \leq \mu_{s}(p, t)\|v\|, \\
\nu_{f}(p, t)^{-1}\|u\| \leq\left\|D \varphi^{-t}(u)\right\| \leq \nu_{s}(p, t)^{-1}\|u\| .
\end{gathered}
$$

Replacing the use of Theorem 1.2 in [Hasselblatt 94b] by Theorem 1.4 gives:

Theorem 4.3. The geodesic flow of a compact bpinched relatively a-pinched Riemannian manifold $N$ is $a-b+\sqrt{(a+b)^{2}+4(1-a) b}+\epsilon$-bunched (and hence, $2 a^{1 /(b+1)}+\epsilon$-bunched) for some $\epsilon>0$.

Using [Hasselblatt 94b, Theorem 5] this implies Theorem 1.6.

\section{EXPLICIT SOLUTIONS AND POLYNOMIAL ASYMPTOTICS}

Explicit solutions of the scalar Riccati equation with monomial forcing are discussed in [Watson 95]. It is easy to check that

$$
u:=\sum_{k=0}^{\infty} \frac{a^{k} \cdot t^{k(n+2)}}{k !(n+2)^{2 k} \Gamma\left(k+\frac{n+1}{n+2}\right)}
$$

solves the Jacobi equation $\ddot{u}=a t^{n} u$, and this implies that $x:=\dot{u} / u$ solves the Riccati equation $\dot{x}+x^{2}=a t^{n}$ (see [Paternain 99, page 37] or [Davis 90]). However, asymptotic ratios can be found without using the given series. In fact, the following result is shown in [Maric 00, Theorem 1.7] and part a) of its proof:

Proposition 5.1. If $f$ solves $\dot{x}+x^{2}=P(t)$ and $f(0) \geq 0$, where $P(t)$ is a polynomial with $P(t)>0$ for $t>$ 0 , then $\lim _{t \rightarrow \infty} f(t)(P(t))^{-1 / 2}=1$. In particular, if $P(t)=a t^{n}$, for $n$ a positive integer and $a>0$, then $\lim _{t \rightarrow \infty} f(t) a^{-1 / 2} t^{-n / 2}=1$.

The result in [Marić 00] applies more generally, where $P(t)$ is replaced by any differentiable function $g(t)$ that is positive for $t>0$ and satisfies $\lim _{t \rightarrow \infty} g^{\prime}(t) /(g(t))^{3 / 2}=0$. Moreover, as shown in [Marić 00], the limit condition on $g$ can be replaced by the weaker assumption that $g^{-1 / 2}$ is Beurling slowly varying (defined on page 7 of [Marić 00]). The proof in [Marić 00] is based on prior work in [Geluk and de Haan 87] and [Omey 97]. The special case of $\dot{x}+$ $x^{2}=a t^{n}$ was known even earlier. For example, it can be treated by applying methods in [Bellman 53, Chapter 6, Sections 3 and 13]. This involves a transformation that goes back to Liouville. See [Bellman 53] for additional bibliographic information.

An immediate consequence of Proposition 5.1 is:

Proposition 5.2. If $P(t)$ is a polynomial with $P(t)>0$ for $t>0$, then the solutions $f_{a}(t)$ of $\dot{x}+x^{2}=a P(t)$ with $f_{a}(0) \geq 0$ satisfy $\lim _{t \rightarrow \infty} f_{a}(t) / f_{1}(t)=\sqrt{a}$.

\section{NUMERICAL WORK AND OPEN QUESTIONS}

Our investigations began by probing for optimality of Theorem 1.2 using solutions of the Riccati equation with $k(t)=e^{\sin t+\sin \pi t+\sin \sqrt{2} t} \in\left(e^{-3}, e^{3}\right)$ on the right-hand side (chosen for being bounded, bounded away from 0 , and "stochastic"). For two solutions $x_{1}(t)$ and $x_{2}(t)$ of the Riccati equation with right-hand side $k(t)$ and $a k(t)$, respectively, and with $x_{1}(0)=x_{2}(0)=1$, we checked 


$$
k(t)=e^{\sin t+\sin \pi t+\sin \sqrt{2} t}
$$$$
x_{2}(t) / x_{1}(t) \text { for } a=1 / 2
$$
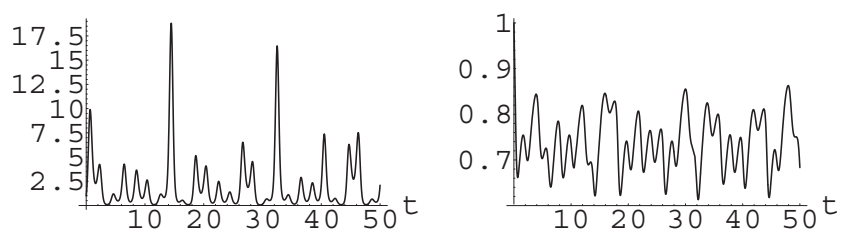

FIGURE 1. Solution ratio for quasiperiodic right-hand side.

whether $\sqrt{a} \leq x_{2}(t) / x_{1}(t)$ for large $t$ using Mathematica. Figure 1 shows that this is not so, and the same fluctuations persist for times around $t=5000$. Therefore, one cannot replace $\sqrt{a}$ by $a$ in (1-1) and Theorem 1.2 is sharp in that sense.

On the other hand, the ratio of solutions stays well above $1 / 2$, which means that the lower bound might be improved over Theorem 1.2. By varying $a$, we computed the function $R_{k}(a):=\inf _{t \geq 0} x_{2}(t) / x_{1}(t)$. We first ob-

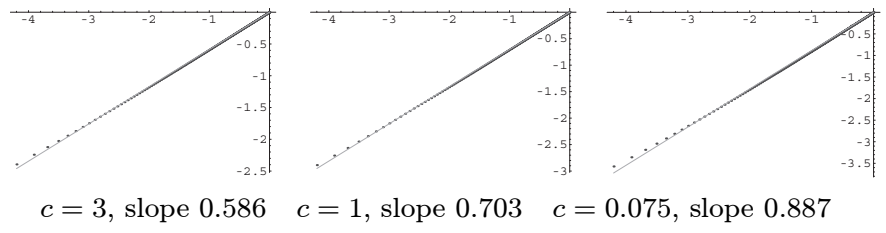

FIGURE 2. Solution ratios versus "spread" parameter $a$ (log-log plot).

tained the log-log plot in the middle of Figure 2, which suggests that the optimal result should have $R_{k}(a) \gtrsim$ $a^{.703}$. The other two plots in Figure 2, obtained for different $c$ in $k_{c}(t):=e^{(\sin t+\sin \pi t+\sin \sqrt{2} t) / c}$, produce analogous lower bounds of Hölder type, only with different exponents, suggesting that $R_{k_{c}}(a) \approx a^{H\left(k_{c}\right)}$. We computed $H\left(k_{c}\right)$ for various values of $c$. The results (in Figure 3) suggest that $\lim _{c \rightarrow 0} H\left(k_{c}\right)=1$, which led us to Theorem 1.3.

The primary effect of the parameter $c$ in the functions $k_{c}$ above is to affect the ratio between the infimum and

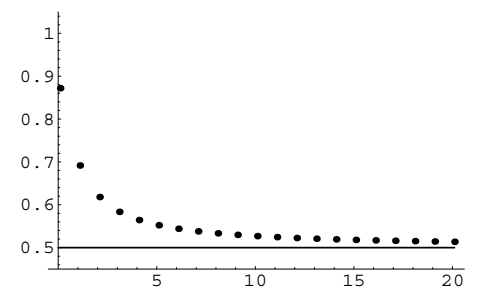

FIGURE 3. $H\left(k_{c}\right)$ versus $c$. supremum of $k_{c}$, i.e., on the absolute bounds on the righthand side. Figure 3 suggests that Theorem 1.2 can be refined by adding hypotheses from Theorem 1.1, i.e., that relative $a$-pinching of the right-hand sides of the Riccati equation combined with absolute $b$-pinching of the righthand sides should force the corresponding solutions to be $a^{H}$-bunched for some $H \in[1 / 2,1]$. This led us to Theorem 1.4.

With Theorem 1.4 in hand, the computations that led to Theorem 1.3 allow us also to test whether Theorem 1.4 is sharp by comparing the actual pinching of the numerical solutions given by the function $R_{k_{c}}(a)$ in Figure 2 with the predicted pinching $B(a, b)$. This is motivated by Figure 1, which shows the solution ratio staying well above $1 / 2$ even though there is only quite weak absolute pinching. Since $k_{c}(t)=e^{(\sin t+\sin \pi t+\sin \sqrt{2} t) / c}$ gives $b=a \inf _{t} k_{c}(t) / \sup _{t} k_{c}(t)=a e^{-6 / c}$, Figure 4 plots $B\left(a, a e^{-6 / c}\right) / R_{k_{c}}(a) \leq 1$ versus $a$ and $c$. If Theorem 1.4

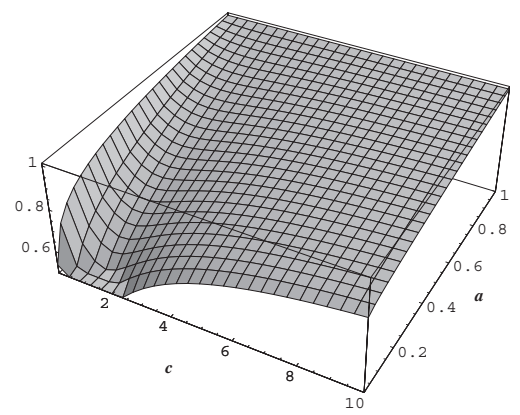

FIGURE 4. Pinching comparison.

were strictly optimal, one would expect the ratio to be essentially 1 for all values of $a$ and $c$. However, for small values of $c$ (which correspond to extremely small values of $b$, i.e., very weak absolute pinching) the computed ratio is as low as about $1 / 2$, and it is truly close to 1 only for strong relative pinching $(a \approx 1)$, which suggests that either the result is yet amenable to further improvement or that quasiperiodic right-hand sides help pinch solutions. One might suspect numerical difficulties for small $c$, but the deviation from 1 is noticeable, albeit small, even for moderate values of $c$ and $a$.

Theorem 1.3 (or even sharpness of any improvements to Theorem 1.4) does not imply that the corresponding differential-geometric results are sharp. The main theorem of [Hasselblatt 94b] uses Theorem 1.2, but Theorem 1.3 does not imply that the result in [Hasselblatt $94 \mathrm{~b}]$ is sharp. It is a subtle differential-geometric question how big the difference between absolute and relative curvature pinching can really be. In Theorem 1.4, we allow 
$a-b$ to be arbitrarily close to 1 , but there might be obstructions to such large separation of the pinching factors for Riemannian metrics (such as in Theorem 1.6). Such obstructions could lead to improvements of the last two results here. On the other hand, it seems likely that $a / b$ can be arbitrarily large. Verifying this would require the construction of examples, for instance, of metrics such that for some orbits of the geodesic flow, the Riccati equation along them has forcing terms on the right-hand side as used in the proof of Theorem 1.3. Ergodicity of the 2 -frame flow might help here.

\section{REFERENCES}

[Anosov and Sinai 67] Dmitriu Viktorovich Anosov and Yakov Sinai. "Some Smooth Ergodic Systems." Russian Mathematical Surveys 22: 5 (1967), 103-167.

[Bellman 53] Richard Bellman. Stability Theory of Differential Equations. New York-Toronto-London: McGrawHill, 1953.

[Bittanti et al. 91] Sergio Bittanti, Alan J. Laub, and Jan C. Willems. The Riccati equation. Berlin: SpringerVerlag, 1991

[Davis 90] Harold T. Davis. Introduction to Nonlinear Differential and Integral Equations. New York: Dover, 1990.

[Fenichel 72] Neil Fenichel. "Persistence and Smoothness of Invariant Manifolds for Flow." Indiana University Mathematics Journal 21 (1971/1972), 193-226; "Asymptotic Stability with Rate Conditions." Indiana University Mathematics Journal 23 (1974), 11091137; $26: 1$ (1977), 81-93; "Hadamard's Generalization of Hyperbolicity, with Applications to the Hopf Bifurcation Problem." In Dynamical Systems, Theory and Applications (Rencontres, Battelle Res. Inst., Seattle, WA, 1974), pp. 525-538, Lecture Notes in Physics 38. Berlin-New York: Springer-Verlag 1975.

[Geluk and de Haan 87] Jaap L. Geluk and Laurens de Haan. Regular Variation, Extensions and Tauberian Theorems, CWI Tract 40. Amsterdam: Stichting Mathematisch Centrum, Centrum voor Wiskunde en Informatica, 1987.

[Hasselblatt 94a] Boris Hasselblatt. "Regularity of the Anosov Splitting and of Horospheric Foliations."

Marlies Gerber, Department of Mathematics, Indiana University, Rawles Hall, Bloomington, IN 47405

(Gerber@swift.math.indiana.edu)

Boris Hasselblatt, Department of Mathematics, Tufts University, Medford, MA 02155 (Boris.Hasselblatt@tufts.edu)
Ergodic Theory and Dynamical Systems 14:4 (1994), 645-666.

[Hasselblatt 94b] Boris Hasselblatt. "Horospheric Foliations and Relative Pinching." Journal of Differential Geometry 39:1 (1994), 57-63.

[Hasselblatt and Wilkinson 99] Boris Hasselblatt and Amie Wilkinson. "Prevalence of Non-Lipschitz Anosov Foliations." Ergodic Theory and Dynamical Systems 19:3 (1999), 643-656.

[Hirsch and Pugh 75] Morris Hirsch amd Charles C. Pugh. "Smoothness of Horocycle Foliations." Journal of Differential Geometry 10 (1975), 225-238.

[Hirsch et al. 77] Morris Hirsch, Charles C. Pugh, and Michael Shub. Invariant Manifolds. Lecture Notes in Mathematics 583. Berlin: Springer-Verlag, 1977.

[Hopf 39] Eberhard Hopf. "Statistik der geodätischen Linien in Mannigfaltigkeiten negativer Krümmung." Berichte über die Verhandlungen der Sächsischen Akademie der Wissenschaften zu Leipzig, Mathematisch-Physikalische Klasse 91 (1939), 261-304.

[Katok and Hasselblatt 95] Anatole Katok and Boris Hasselblatt. Introduction to the Modern Theory of Dynamical Systems. Encyclopedia of Mathematics and Its Applications 54. Cambridge, UK: Cambridge University Press, 1995.

[Klingenberg 82] Wilhelm Klingenberg. Riemannian Geometry. Berlin: de Gruyter, 1982.

[Marić 00] Vojislav Marić. Regular Variation and Differential Equations. Lecture Notes in Mathematics 1726. Berlin: Springer-Verlag, 2000.

[Omey 97] Edward Omey. "Rapidly Varying Behaviour of the Solutions of a Second Order Linear Differential Equation." In Proceedings of the Seventh International Colloquium on Differential Equations, pp. 295-303. Utrecht: VSP, 1997.

[Paternain 99] Gabriel Pedro Paternain. Geodesic Flows. Progress in Mathematics 180. Boston-Basel-Berlin: Birkhäuser, 1999.

[Watson 95] George Neville Watson. A Treatise on the Theory of Bessel Functions. Cambridge, UK: Cambridge University Press, 1922, 1944, 1966, 1995.

Daniel Keesing, Department of Mathematics, Tufts University, Medford, MA 02155 (Daniel.Keesing@tufts.edu)

Received June 27, 2002; accepted in revised form June 25, 2003. 